\title{
Structure and dynamics of water in nonionic reverse micelles: A combined time-resolved infrared and small angle $x$-ray scattering study
}

\author{
Tibert H. van der Loop, ${ }^{1, a)}$ Matthijs R. Panman, ${ }^{1}$ Stephan Lotze, ${ }^{2}$ Jing Zhang, ${ }^{3}$ \\ Thomas Vad, ${ }^{4}$ Huib J. Bakker, ${ }^{2}$ Wiebke F. C. Sager, ${ }^{3, b)}$ and Sander Woutersen ${ }^{1, c)}$ \\ ${ }^{1}$ Van 't Hoff Institute for Molecular Sciences, University of Amsterdam, Science Park 904, \\ 1098 XH Amsterdam, The Netherlands \\ ${ }^{2}$ FOM Institute for Atomic and Molecular Physics, Science Park 104, 1098 XG Amsterdam, The Netherlands \\ ${ }^{3}$ Institute of Complex Systems-Forschungszentrum Juelich, 52425 Juelich, Germany \\ ${ }^{4}$ Institut für Textiltechnik, RWTH Aachen University, Otto-Blumenthal-Str. 1, 52074 Aachen, Germany
}

(Received 16 April 2012; accepted 26 June 2012; published online 26 July 2012)

\begin{abstract}
We study the structure and reorientation dynamics of nanometer-sized water droplets inside nonionic reverse micelles (water/Igepal-CO-520/cyclohexane) with time-resolved mid-infrared pumpprobe spectroscopy and small angle $\mathrm{x}$-ray scattering. In the time-resolved experiments, we probe the vibrational and orientational dynamics of the O-D bonds of dilute $\mathrm{HDO}: \mathrm{H}_{2} \mathrm{O}$ mixtures in Igepal reverse micelles as a function of temperature and micelle size. We find that even small micelles contain a large fraction of water that reorients at the same rate as water in the bulk, which indicates that the polyethylene oxide chains of the surfactant do not penetrate into the water volume. We also observe that the confinement affects the reorientation dynamics of only the first hydration layer. From the temperature dependent surfacewater dynamics, we estimate an activation enthalpy for reorientation of $45 \pm 9 \mathrm{~kJ} \mathrm{~mol}^{-1}$ $\left(11 \pm 2 \mathrm{kcal} \mathrm{mol}^{-1}\right)$, which is close to the activation energy of the reorientation of water molecules in ice. () 2012 American Institute of Physics. [http://dx.doi.org/10.1063/1.4736562]
\end{abstract}

\section{INTRODUCTION}

Water confined in nanometer-sized volumes can be found in many biological systems. Well-known examples include water between lipid layers ${ }^{1}$ and in protein pumps. ${ }^{2,3}$ Understanding the effect of confinement on the properties of water is crucial in explaining the function of certain proteins. ${ }^{4}$ This can be understood by the fact that the structure and dynamics of proteins are strongly coupled to the dynamics of the surrounding water molecules. Reverse micelles, self-assembled structures of isolated surfactant coated water droplets, are a convenient model system for studying water in nanoconfinement. ${ }^{5}$ Furthermore, reverse micelles can promote the synthesis of nearly monodisperse nanoparticles of controlled size. ${ }^{6,7}$ In some examples of nanoparticle synthesis, the use of reverse micelles even allows for the control of crystal structure. ${ }^{8}$ These remarkable properties additionally stress the relevance of studying these versatile systems.

The addition of water to a two-component reverse micellar system, i.e., surfactant self-assemblies in an apolar solvent, leads to an uptake of water molecules in the hydrophilic interior of the reverse micelles and finally to thermodynamically stable water-in-oil (w/o) microemulsions, in which nanosized water droplets are stabilized by a saturated surfactant monolayer. The properties of these systems, such as water droplet size, shape, polydispersity and droplet interaction, as well as

\footnotetext{
a)Electronic mail: T.H.vanderLoop@uva.nl.

b) Electronic mail: W.Sager@fz-juelich.de.

c)Electronic mail: S.Woutersen@uva.nl.
}

their single phase existence regions, depend besides composition mainly on the elastic properties of the interfacial surfactant layer. These elastic properties have been described in terms of a preferred curvature (radius of spontaneous curvature) and film stiffness (rigidity constants for mean and Gaussian curvature). They are, for a given surfactant and oil, influenced by parameters such as temperature and/or salt concentration of the aqueous phase. ${ }^{7,-13}$ In principle, spherical water droplets exist over a certain range of composition and formulation variable space. Their size has been found to depend linearly on the ratio between water and surfactant and can thus be easily tuned, where the molar ratio is commonly defined as

$$
w_{\circ}=\frac{\left[\mathrm{H}_{2} \mathrm{O}\right]}{[\text { Surfactant }]} .
$$

However, one should keep in mind that if the radius of the spherical droplets does not correspond to the radius of spontaneous curvature, the droplets are subject to size and/or shape transformation that will finally lead to phase separation. If the radius of spontaneous curvature is smaller than the droplet radius, the droplets will shrink by expelling water into an excess water phase (solubilization boundary limit). If the interfacial film is less curved toward water, the droplets will adapt to the preferred curvature by changing their interaction from repulsive to attractive ${ }^{11,12}$ and/or by forming more and longer cylinders, ${ }^{13}$ whereby interconnected cylindrical networks will separate into a bilayer phase.

Reverse micellar systems can be prepared with ionic or nonionic surfactants. The type of surfactant used determines 
the overall properties of the ternary surfactant system. For instance, ionic and nonionic microemulsions reveal an opposite phase behavior with temperature. The dominant factor that influences the properties of the interfacial surfactant layer is temperature for nonionic surfactants and salt concentration for ionic surfactant systems. ${ }^{14}$ Ionic reverse micelles or w/o microemulsions have been more extensively investigated than nonionic reverse micelles. However, it is important to realize that ionic reverse micelles intrinsically carry a large counterion concentration, which decreases with increasing amount of added water and is thus inversely proportional to $w_{\circ}$. For instance, an ionic reverse micelle with $w_{\circ}=5$ has a counterion concentration as high as 11 molal (0.56 molal at $w_{\circ}=100$ ), reaching salt concentrations that are known to drastically affect the dynamics of water. ${ }^{15,16}$ In ionic reverse micelles the effect of confinement on the dynamics of water thus cannot be independently studied, especially for small $w_{\circ}$.

For nonionic surfactants with, e.g., an ethylene glycol (ethylene oxide or oxyethylene) chain as the hydrophilic moiety, the water in the reverse micellar interior is not perturbed by charges. In general, the (complex) temperature dependence of the interaction between water and ethylene oxide is responsible for the rich phase behavior that this type of nonionic surfactant system displays. ${ }^{17}$

There are numerous techniques available to study different properties of reverse micellar systems. The size and structure of reverse micelles can be resolved with neutron ${ }^{18}$ and $\mathrm{x}$ ray ${ }^{19,20}$ scattering. Terahertz spectroscopy in reverse micelles has revealed the low vibrational modes characteristic for confined water. ${ }^{21}$ These modes are size-dependent and support the idea of a behavior specific to water in nanoconfinement. NMR has also proven to be a valuable tool for measuring the effect of confinement on the rotational dynamics by means of a molecular probe. ${ }^{22}$ Probe molecules are also used in optical techniques. ${ }^{23,24}$ Optical techniques have the advantage of having long lived excited-states, thus creating a large time window for dynamic measurements. However, the difficulty with using molecular probes lies in determining where the molecule is located inside the reverse micelle, thus making it challenging to determine which part of the water in the reverse micelle is being probed. ${ }^{25}$

The rotational dynamics of water molecules can be observed using time-resolved polarization-dependent vibrational pump-probe spectroscopy. This is achieved by measur-

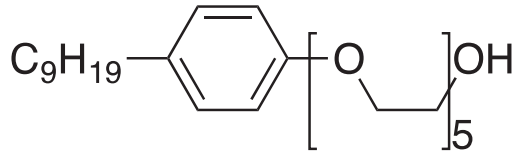

FIG. 1. Igepal CO-520 surfactant molecule, pentaoxyethylene nonylphenyl ether. The alkyl chain is branched.

ing the anisotropy decay of the OD- or OH- stretch vibration. Terahertz studies have shown that reorientation of water is isotopically invariant. ${ }^{26}$ The reorientation of water molecules in ionic reverse micellar systems has previously been studied by measuring the anisotropy decay for different surfactants and micelle sizes. ${ }^{27-32}$ These studies have revealed that water in reverse micelles can be well described by a twospecies model that is composed of "free" water that behaves like water in the bulk, and water that resides on the inner surface of the reverse micelles. For example, in sodium bis(2ethylhexyl) sulfosuccinate reverse micelles the reorientation time of the surface-bound water molecules at room temperature was found to be much slower, $>16 \mathrm{ps},{ }^{27,30}$ than that of bulk water, which is around 2.5 ps. $^{33,34,46}$

In nonionic reverse micelles, similar observations have been made. ${ }^{30-32}$ Water, in this case, can also be described by a surface and a core component. Although these studies nicely show a similarity between ionic and nonionic systems, ${ }^{30}$ a comprehensive study of the size and temperature dependence of the water dynamics in nonionic reverse micelles is still lacking. In this paper, we study the size and temperature dependence of the structure and dynamics of Igepal (see Figure 1) reverse micelles by combining small angle $\mathrm{x}$-ray scattering (SAXS) measurements and time-resolved infrared spectroscopy.

\section{MATERIALS AND METHODS}

The SAXS measurements were performed at the European Molecular Biology Laboratory (EMBL) X33 beamline at the Doris III storage ring (Deutsches Elektronen Synchrotron (DESY), Hamburg, Germany). The reverse micellar samples were filled into a $100 \mu \mathrm{L}$ thermostated flat in-air cell with mica windows. The composition and temperature of the samples are listed in Table I. The data were recorded between 293 and $320 \mathrm{~K}$ using a $1 \mathrm{M}$ Pilatus detector (DECTRIS) at a sample-to-detector distance of $2.7 \mathrm{~m}$ and a wavelength of

TABLE I. Structure parameters obtained from the refinement of the SAXS scattering curves using a multicomponent model for hard spheres at different $w_{\circ}$ and temperature. The reverse micelles are characterized by the droplet radius $r_{\circ}$, polydispersity parameter $\sigma_{\circ}$ of the log-normal size distribution and droplet concentration $c_{\circ}$. The surface to surface distance $D_{\diamond}$ corresponds to twice the shell thickness built up by the surfactant tails. $c_{w}$ is the mass fraction of water plus surfactant.

\begin{tabular}{ccccccc}
\hline \hline$w_{\circ}$ & 5 & 8.6 & 12.3 & 18.4 & \multicolumn{2}{c}{5} \\
$c_{w}$ & 0.159 & 0.175 & 0.191 & 0.216 & 313 & 0.159 \\
$\mathrm{~T}(\mathrm{~K})$ & & & 303 & & 320 \\
\hline$c_{\circ}\left[10^{-4} \mathrm{~nm}^{-3}\right]$ & $4.562(1)$ & $3.105(1)$ & $2.249(1)$ & $1.107(1)$ & $4.196(1)$ & $3.748(1)$ \\
$r_{\circ}[\mathrm{nm}]$ & $2.91(1)$ & $3.66(1)$ & $4.27(1)$ & $5.79(1)$ & $3.03(1)$ & $3.12(1)$ \\
$\sigma_{\circ}$ & $0.177(1)$ & $0.168(1)$ & $0.130(1)$ & $0.145(2)$ & $0.174(1)$ & $0.177(2)$ \\
$D_{\circ} / 2[\mathrm{~nm}]$ & & \multicolumn{7}{c}{$0.91(1)$} \\
\hline \hline
\end{tabular}


$0.15 \mathrm{~nm}$, covering a range of momentum transfer of $0.1 \leq q$ $\leq 4 \mathrm{~nm}^{-1}(q=4 \pi \sin (\theta) / \lambda$ is the modulus of the scattering vector, $2 \theta$ is the scattering angle and $\lambda$ is the wavelength). After normalization to the intensity of the transmitted beam the collected data were radially averaged. ${ }^{35}$ The solvent was subtracted prior to data analysis.

The SAXS data were fitted with a model function based on Vrij's analytical solution for a multicomponent system of hard spheres. ${ }^{36-38}$ Since the electron densities of water and ethylene glycol as well as of the nonylphenol tail and cyclohexane are similar, the part of the spherical reverse micelles that contributes to the scattering is the headgroup shell and interior water, while the tail shell is invisible to x-rays. The structure parameters characterizing the system are the concentration $c_{\circ}$, the radius $r_{\circ}$, the polydispersity parameter $\sigma_{\circ}$ of the log-normal size-distribution and the surface to surface distance $D_{\circ} . D_{\circ} / 2$ is equal to the thickness of the hydrophobic layer around the reverse micelle, see Figure 12. The scattering curves are carefully checked to exclude attractive interactions and contributions stemming from cylindrical scatterers, especially at low temperatures and higher $w_{\circ}$.

All time-resolved measurements were carried out with an amplified Ti:Sapphire system (Coherent Legend Elite, $800 \mathrm{~nm}, 35 \mathrm{fs}, 3 \mathrm{~mJ}$ ) pumping a commercial (OperA solo) optical parametric amplifier with a difference-frequency generation option to produce a pulse of $\sim 20 \mu \mathrm{J}$ energy at $2500 \mathrm{~cm}^{-1}$ and $150 \mathrm{~cm}^{-1}$ bandwidth. The cross-correlation of the pump and probe pulse in InAs shows a FWHM of 150 fs. The polarization-dependent vibrational dynamics of the OD-stretch of $10 \%$ HDO in water in reverse micelles was measured by exciting the samples to the $v=1$ state of the OD-stretch mode using a mid-IR pulse resonant with the ODstretch vibration. A probe pulse of around $4 \%$ of the pump energy measures the time-dependent transient absorption after a variable delay time $t$. To exclude long time effects due to heating the samples were circulated with a Verder 2030 pump. The IR light from the probe beam and a reference beam were detected after passing through the sample with a custom-made liquid nitrogen cooled 32 pixel $\mathrm{HgCdTe}$ array detector.

The anisotropy was constructed from the parallel and perpendicular time-dependent transient absorption. The anisotropy decays due to the orientational randomization of excited OD-stretch oscillators, ${ }^{39}$ and thus directly reveals the reorientation dynamics of the water molecules. At long time delays, a small residual signal in the absorption difference, associated with heating of the sample by the pump pulse, remains. This heating effect was subtracted from the parallel and perpendicular transient response before calculating the anisotropy. The heating effect was assumed to grow in to a final level with the same delay dependence as the population relaxation. The difference between the parallel and perpendicular signal, after normalization, is the anisotropy of the excited vibration,

$$
R(v, t)=\frac{\Delta \alpha_{c}(v, t)_{\|}-\Delta \alpha_{c}(v, t)_{\perp}}{\Delta \alpha_{c}(\nu, t)_{\|}+2 \Delta \alpha_{c}(v, t)_{\perp}},
$$

where $v$ is the probe frequency, $\Delta \alpha_{c}(\nu, t)_{\|}$and $\Delta \alpha_{c}(v, t)_{\perp}$ are time-dependent parallel and perpendicular transient absorption changes corrected for the ingrowing heat signal.

The rotation-free signal was constructed from the parallel and perpendicular signals in the following way:

$$
\Delta \alpha_{R F}(v, t)=\frac{\Delta \alpha(v, t)_{\|}+2 \Delta \alpha(v, t)_{\perp}}{3} .
$$

The transient absorption decay of the OD-stretch was measured for a series of reverse micelles. Samples consisting of water, cyclohexane, and Igepal CO-520 had molar water surfactant ratios $w_{o}$ of $2.5,5,8,12.5$, and 15 and were prepared by a combination of pipetting and weighing. The samples were prepared such that cyclohexane (spectroscopic grade) always amounted 0.8 to the total mass. $\mathrm{D}_{2} \mathrm{O}(99.9 \%)$ was ordered from Eurisotop, all other chemicals were purchased from Sigma-Aldrich. All samples were prepared with demineralized Milli-Q water, stored at $6^{\circ} \mathrm{C}$ and measured within a week after preparation.

The temperature was controlled to within $1 \mathrm{~K}$ using a TE TC-48-20 thermostat in combination with heat rods connected to the sample. The temperatures in the sample cell were calibrated with an infrared camera (FLIR ThermaCAM ${ }^{\circledR}$ E2). Steady-state infrared spectra were acquired on a Bruker Vertex 70 FTIR spectrometer. Reference samples were made with pure $\mathrm{H}_{2} \mathrm{O}$ and subtracted from $\mathrm{HDO}: \mathrm{H}_{2} \mathrm{O}$ micelle spectra to obtain the OD-stretch spectra.

Water fractions $w_{\circ}$ were verified from the ratio between the absorption of the bending mode of $\mathrm{H}_{2} \mathrm{O}$ and two vibrational peaks that belong to Igepal (see Figure 2). The micelle steady-state infrared spectra between 1560 and $1680 \mathrm{~cm}^{-1}$ for different micelle sizes can be well fitted by a weighted sum of the absorption spectra of neat Igepal and water:

$$
A_{\text {micelle }}\left(v, w_{0}\right)=N\left(f\left(w_{\circ}\right) A_{\mathrm{H}_{2} \mathrm{O}}(v)+A_{\text {Igepal }}(v)\right),
$$

where $A$ is the absorbance, $N$ a normalization constant, and $f\left(w_{\circ}\right)$ the ratio absorption of Igepal and $\mathrm{H}_{2} \mathrm{O}$. From a series of spectra with known $w_{\circ}$ we find that $f\left(w_{\circ}\right)$ is directly proportional to $w_{\circ}$,

$$
f\left(w_{\circ}\right)=k w_{\circ},
$$

with $k=0.078(3)$. The calibration line was used to verify $w_{\circ}$ in situ.
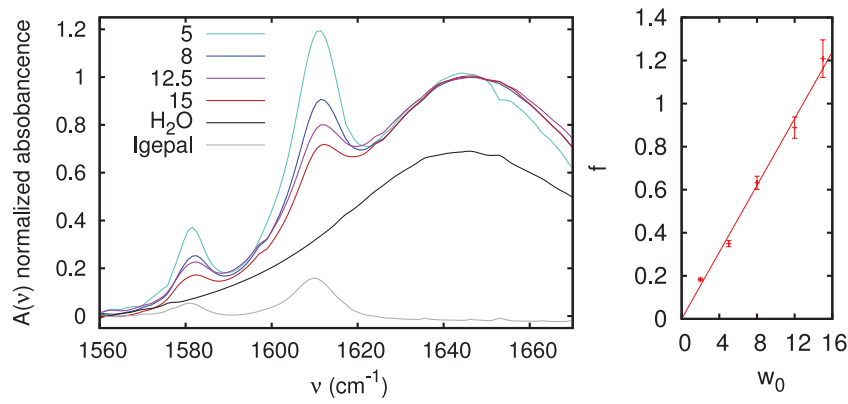

FIG. 2. Left: Steady-state absorption of reverse micelles of different size, neat Igepal and $\mathrm{H}_{2} \mathrm{O}$. Right: Calibration line that allows for an estimation of $w_{\circ}$ from the steady-state infrared spectrum. The slope $a$ is found to be $0.078(3)$. 


\section{RESULTS AND DISCUSSION}

Figure 3 displays SAXS curves for reverse micelles with a $w_{\circ}$ of $5,8.6,12.3$, and 18.4 at $303 \mathrm{~K}$. The shape of the scattering curves clearly indicates the spherical nature of the reverse micelles. With increasing $w_{\circ}$ the minimum shifts to smaller scattering vectors $q$ revealing larger droplet sizes. This is also reflected in an increase in the scattering intensity at low $q$, as the forward scattering scales with the square of the droplet volume. The scattering curves are analyzed using a model function based on Vrij's analytical solution for a multicomponent hard sphere system. ${ }^{36-38}$ The results from the structural refinement are shown in Table I. Since the electron density of the nonylphenyl surfactant tail is similar to that of cyclohexane, the x-rays see only droplets made up of the ethylene oxide head group moiety and the interior water. Plotting the obtained radius $r_{\circ}$, versus $w_{\circ}$ thus gives the length of the ethylene oxide chain $l_{h}$ as intercept (see inset of Figure 3). The value of 1.8(2) nm corresponds to an extended chain (zig-zag) configuration with a length of $3.5 \AA$ per monomer, which is

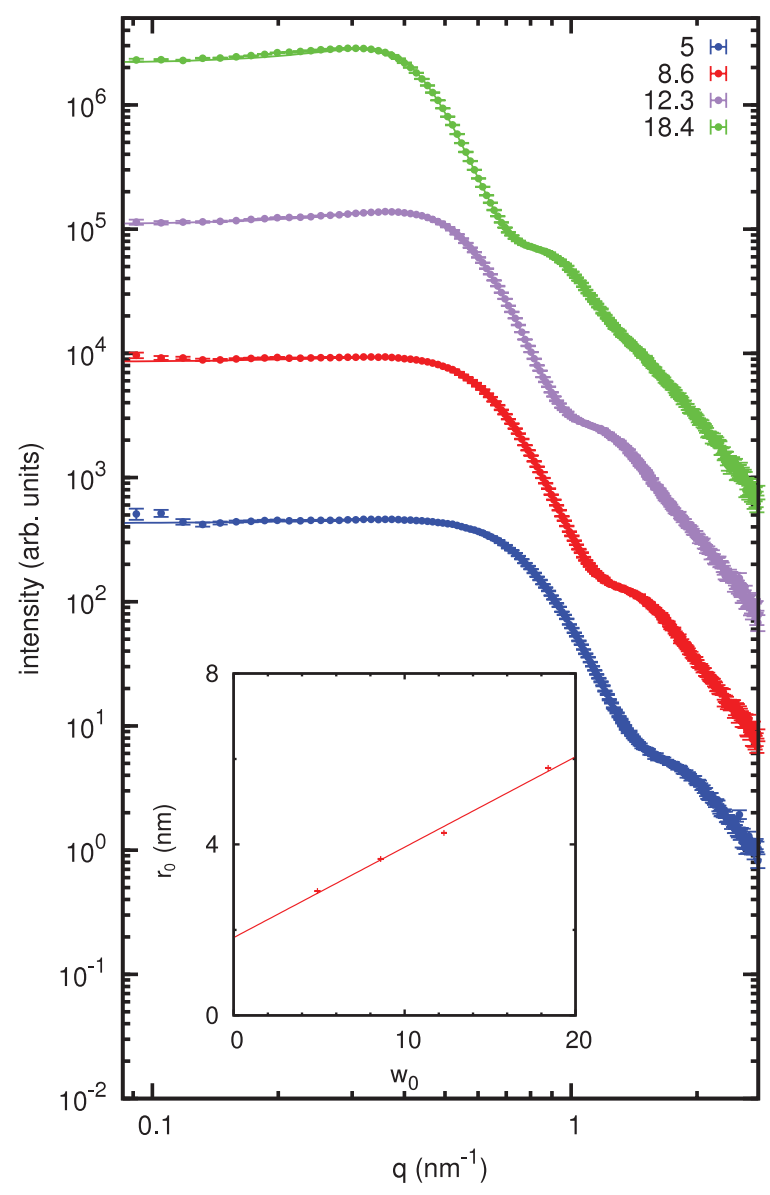

FIG. 3. Small-angle x-ray scattering curves (scattering intensity versus the modulus of the momentum transfer vector $q$ ) from reverse micelles for a $w_{\circ}$ of 5, 8.6, 12.3, and 18.4 at $303 \mathrm{~K}$. Successive scattering curves are displaced upwards by one logarithmic unit for better visualization. The shape of the curves is typical for spherical scatterers. The scattering data were fitted with a multicomponent model for hard spheres. The fits are shown as solid lines and the refined parameters are presented in Table I. The inset displays the linear dependence of the radius $r_{\circ}$ on $w_{\circ}$. Linear regression (red line) gives the proportionality factor $(a=0.21(1) \mathrm{nm}$ ) as the slope and the length of the ethylene oxide chain (head group shell thickness $l_{h}=1.8(2) \mathrm{nm}$ ) as the intercept. expected for short ethylene oxide chains. ${ }^{40}$ From the slope, the proportionality factor $a$ for the linear scaling of the reverse micelle radius with $w_{\circ}$ is obtained, resulting in $a=0.21(1) \mathrm{nm}$. The length of the hydrophobic surfactant tail is retrieved from the half of the droplet surface to surface distance $D_{\circ}\left(D_{\circ} / 2=0.91(1) \mathrm{nm}\right)$, see Figure 12. For reverse micelles with $w_{\circ}=5$, the temperature dependence of the structural parameters has been investigated. With increasing temperature the spherical droplets increase slightly in size, while the droplet concentration decreases (see Table I). This can be explained by an increased monomer concentration of the surfactant in cyclohexane at higher temperature, leaving less surfactant for stabilizing the interface between water and oil. At lower temperatures, below $298 \mathrm{~K}$, an attractive interaction between the droplets has to be taken into account (sticky hard sphere potential ${ }^{37}$ ).

Figure 4 shows the OD-stretch absorption spectrum of $10 \%$ deuterated Igepal CO-520 and HDO in water for several reverse micelle sizes. Part of the infrared absorption in the reverse micelles arises from the OD-group of the surfactant. As reported before, ${ }^{41}$ when decreasing the micelle size, the peak maximum shifts to higher frequencies. This can be explained by a weakening of the hydrogen bond network ${ }^{42}$ and will be further discussed in the context of the time-resolved data.

Figure 5 shows a typical isotropic time-dependent transient absorption spectrum of a $w_{o}=5$ sample for several time delays with respect to the pump pulse. The spectrum shows a positive and a negative feature. The negative absorption change on the blue side of the spectrum is a result of depopulation of the $v=0$ state and $v=1 \rightarrow 0$ stimulated emission. The positive absorption change on the red side of the spectrum is a result of induced absorption of the $v=1 \rightarrow v=2$ state. The signal decays on a picosecond time scale due to vibrational relaxation. A small residual offset is observed at long time delays, and is due to a pump-induced heating of the sample. ${ }^{43}$ The effect is small compared to the total signal, and is much smaller than it is in bulk water. ${ }^{34}$ This difference in the heating effect is due to the large volume available for dissipation of heat in the reverse micellar samples: the water fraction in reverse micelles is essentially diluted by cyclohexane. This results in a lower energy density upon vibrational excitation and al-

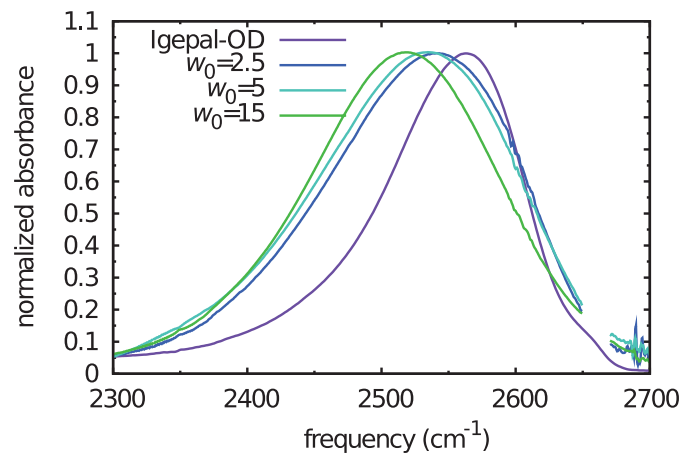

FIG. 4. The OD-stretch absorption spectrum of HDO: $\mathrm{H}_{2} \mathrm{O}$ in Igepal reverse micelles for different $w_{o}$ values, and the OD-stretch spectrum of neat isotopically dilute Igepal CO-520, all recorded at $303 \mathrm{~K}$. The micelle spectra were obtained by subtracting a reference micelle solution of pure $\mathrm{H}_{2} \mathrm{O}$. 


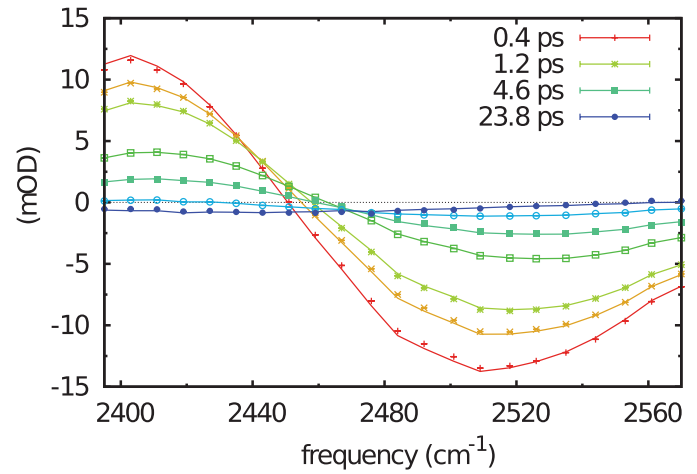

FIG. 5. Isotropic transient spectrum of the OD-stretch at different time delays in reverse micelles of $w_{\circ}=5$ at $303 \mathrm{~K}$. Solid lines are the results of the global fit described in the the text.

lows for a more effective dissipation of the energy compared to water in the bulk. As can be seen in Figure 6, the rise and decay of the delay scans at 2442 and $2458 \mathrm{~cm}^{-1}$ clearly show a multi-exponential character. In Figure 7, the normalized transient response is plotted at probe frequency of $2484 \mathrm{~cm}^{-1}$. The logarithmic inset shows that, also at this higher frequency, the response is multi-exponential. For increasing reverse micelle size the contribution of the fast component becomes smaller.

In contrast to earlier studies, ${ }^{30,31}$ our measurements are carried out over a frequency range that extends further into the lower part of the OD-stretch spectrum. At these lower frequencies we observe an additional fast component that is faster than the $T_{1}$ of bulk water. A previous study ${ }^{30}$ assumed Igepal-OD to have a slower $T_{1}$ than that of bulk HDO: $\mathrm{H}_{2} \mathrm{O}$. We argue that, instead of a slow component, the transient response of Igepal-OD is actually this fast component at lower frequencies. First of all, the relative contribution of this fast component depends inversely on the size of a reverse micelle, as can be seen in Figure 7 (and will later on be confirmed by the global analysis of the spectral components, which are plotted in Figure 9). This size-dependence of the fast component is expected for the Igepal-OD response, as the amount of Igepal-OD groups compared to the HDO molecules increase

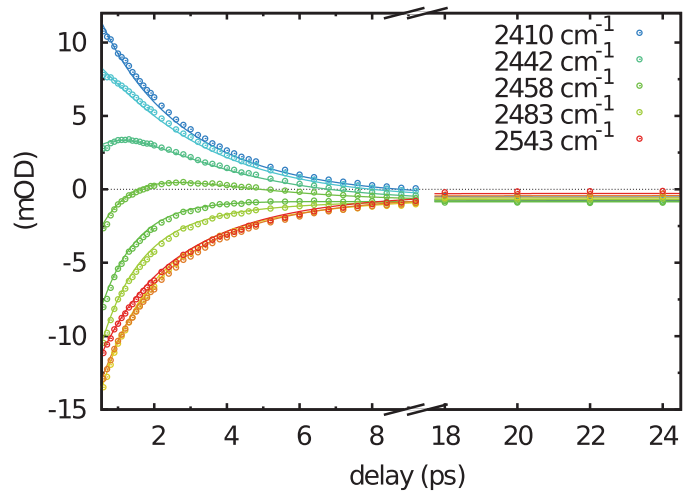

FIG. 6. Isotropic transient decay curves of the OD-stretch for several frequencies for $w_{\circ}=5$ at $303 \mathrm{~K}$. At long time delay, a small residual frequencydependent value is observed. The non-monotonic decay between 2442 and $2458 \mathrm{~cm}^{-1}$ reveals the multi-exponential character of the data. The curves are the result of the global fit described in the text.

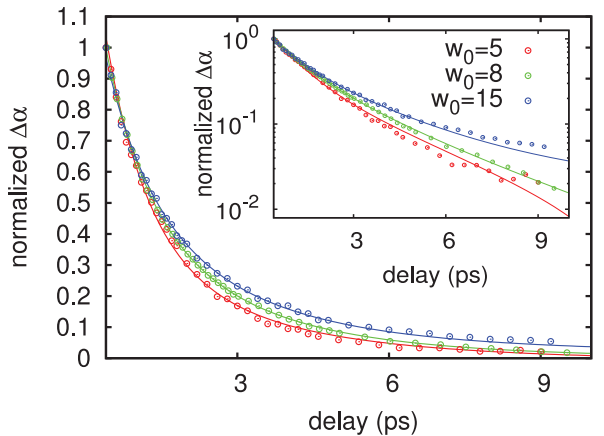

FIG. 7. Normalized transient absorption for reverse micelles of different size at $2484 \mathrm{~cm}^{-1}$ and $303 \mathrm{~K}$ (residual thermal signal subtracted). The inset shows the logarithm of the transient absorption signal.

for smaller reverse micelles. Second, the lifetime associated with the fast component agrees very well with earlier measurements on other alcohols. Ethanol-OD in ethanol-OH has an OD-stretch $T_{1}$ of 800 fs (Ref. 44) and methanol-OD in methanol-OH of $500 \mathrm{fs},{ }^{45}$ both of which agree well with the $810 \mathrm{fs}$ for the fast component that we observe. To confirm our hypothesis that the fast component is due to Igepal, we also measured the transient response of the OD-stretch vibration in neat Igepal, which reveals a similar fast component of $1.2 \mathrm{ps}$.

We model the OD-stretch response of the reverse micelles as the sum of three contributions: ${ }^{30}$ bulk water HDO, surface water HDO, and the OD group of Igepal. Surface water is expected to have a slightly slower $T_{1}$ than bulk water. To reduce the number of free parameters, four simplifying assumptions are made. First, surface and bulk water are assumed to have the same absorption spectrum. Second, temperaturedependent bulk lifetimes for reorientation and population relaxation are used from literature ${ }^{46}$ to describe the bulk component. Third, the bulk fraction $S$ is assumed to be temperature independent. The SAXS measurements reveal that the size of the reverse micelles changes only slightly as a function of temperature (see Table I). Finally, the model includes an offset at long time delays to account for heating. This gives us the following expression for the isotropic absorption change:

$$
\begin{aligned}
\Delta \alpha\left(v, w_{o}, T, t\right)= & A(v, T) e^{-t / T_{1: i}(T)} \\
& +B(v, T)\left[S\left(w_{o}\right) e^{-t / T_{1: b}(T)}\right. \\
& \left.+\left(1-S\left(w_{o}\right)\right) e^{-t / T_{1: s}(T)}\right] \\
& +C(v, T),
\end{aligned}
$$

where $A(v, T)$ and $B(v, T)$ are the size- and temperaturedependent spectra of Igepal-OD and HDO, $C(v, T)$ is the temperature effect, $T_{1: i}(T), T_{1: b}(T)$, and $T_{1: s}(T)$ are temperaturedependent relaxation times of Igepal, bulk water, and surface water, respectively; $S\left(w_{o}\right)$ is the fraction of bulk water. The population relaxation data could be well described by assuming the same rate for the decay of the induced absorption as for the the decay of the bleaching. This is in agreement with previous studies. ${ }^{27,31}$

Having multiple spectrally overlapping species present renders the anisotropy-decay dependent on the vibrational lifetimes. ${ }^{27,30,47}$ However, because we are measuring the 
anisotropy at frequencies at which the contribution of the fast component (Igepal) is very small, and due to the intrinsic fast population relaxation of Igepal-OD, the contribution of Igepal-OD can be neglected. Therefore, the anisotropy decay in the region between 2544 and $2570 \mathrm{~cm}^{-1}$ is analyzed by only two components, surface water and bulk water, yielding the following expression for the anisotropy decay:

$$
\begin{aligned}
R_{\mathrm{HDO}}\left(t, w_{o}, T\right)= & R_{o}\left(v, w_{o}\right) \\
& \times \frac{S\left(w_{o}\right) e^{-t / T_{1: b}(T)-t / \tau_{b}(T)}+\left(1-S\left(w_{o}\right)\right) e^{-t / T_{1: s}(T)-t / \tau_{s}(T)}}{S\left(w_{o}\right) e^{-t / T_{1: b}(T)}+\left(1-S\left(w_{o}\right)\right) e^{-t / T_{1: s}(T)}} .
\end{aligned}
$$

In this equation, $R_{o}\left(v, w_{o}\right)$ is the anisotropy at $t=0$, which theoretically should be 0.4 . $R_{\circ}$ values slightly lower than 0.4 can be observed due to the librational motion of the OD-strech in the first $100 \mathrm{fs} .{ }^{48} \tau_{b}(T)$ and $\tau_{s}(T, v)$ are the temperaturedependent reorientation times of bulk and surface water, respectively. Because the anisotropy decay $R_{\mathrm{HDO}}\left(t, w_{o}, T\right)$ and the isotropic vibrational relaxation $\Delta \alpha\left(v, w_{o}, T, t\right)$ are both dependent on the $T_{1}$ 's of surface and bulk water, the anisotropy and population relaxation were analyzed together in a single global fit. We find that in order to obtain a proper fit, the surface water reorientation time must be allowed to be size-dependent. All time constants were treated as global parameters over the measured frequency range. All time constants except for the surface reorientation were assumed to be size independent. The bulk water fraction $S$ was assumed to be temperature-independent.

Figure 8 shows the experimentally observed anisotropy decay and the global fit for three different $w_{\circ}$ 's at $303 \mathrm{~K}$, and for $w_{\circ}=5$ at three different temperatures: 303, 313, and $323 \mathrm{~K}$. The time constants, bulk water fractions, and the anisotropy at $t=0$ obtained from the global fit are shown in Table II.

The frequency-dependent pre-factors can be plotted as difference absorption spectra of the individual contributions at $t=0$. The spectra of the contributions of the Igepal-OD, HDO, and the heat at $t=\infty$ effect are plotted in Figure 9 as $A(v), B(v)$, and $C(v)$, respectively. Note that the relative Igepal

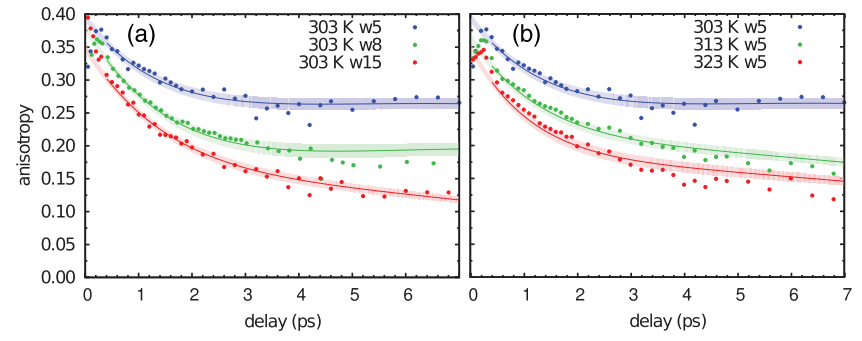

FIG. 8. Anisotropy decay $R_{\mathrm{HDO}}$ for reverse micelles of different size, (a) $w_{o}=5,8$, and 15 at $303 \mathrm{~K}$, and temperature, (b) $w_{o}=5$ at 303, 313, and $323 \mathrm{~K}$. The plotted anisotropy is the average anisotropy between 2544 and $2570 \mathrm{~cm}^{-1}$. The corresponding fit curves from the global analysis are plotted as solid lines. The shaded region represents the standard deviation of the fit function. We observe heteroscedasticity in the anisotropy because the signal gets smaller due to vibrational relaxation. contribution (compared to HDO) increases for decreasing reverse micelle size. This confirms our assignment of the fast component to the Igepal OD-stretch.

Interestingly, we find that the temperature-dependence of the anisotropy can only be fitted if the surface water reorientation time constant $\tau_{s}$ is allowed to change with the size of

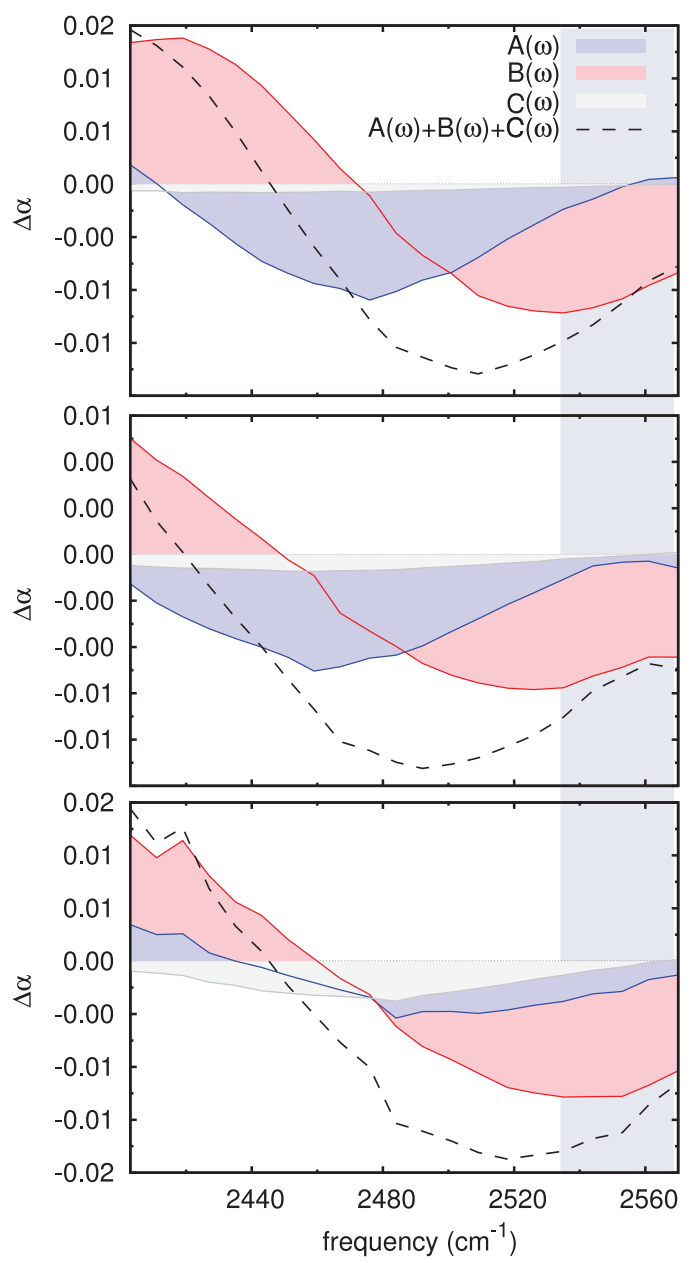

FIG. 9. Spectra of the fast, slow, and the residual signal amplitudes $A(v)$, $B(v)$, and $C(v)$, respectively, plotted for increasing reverse micelle size. Relatively, $B(v)$ decreases with reverse micelle size and $A(v)$ increases with reverse micelle size. The dashed line is the sum of all three contributions. The shaded region at high frequencies indicates the frequency range that is used to construct the anisotropy of Figure 8 . 
TABLE II. Fit results from globally fitting the size and temperature dependence of the isotropic vibrational decay and the anisotropy. The bulk $T_{1}, T_{1: \mathrm{b}}$ and the bulk reorientation time $\tau_{\mathrm{b}}$ are taken from bulk measurements. ${ }^{46}$ The $T_{1: i}$ and $T_{1: s}$ are the $T_{1}$ 's of surfactant and surface water, respectively; $S$ is the fraction of HDO that behaves as bulk water and $R_{\circ}$ is the anisotropy at $t=0$.

\begin{tabular}{ccccccccc}
\hline \hline $\mathrm{T}(\mathrm{K})$ & $w_{o}$ & $T_{1: \mathrm{b}}(\mathrm{ps})($ Ref. 46) & $\tau_{\mathrm{b}}(\mathrm{ps})($ Ref. 46) & $T_{1: \mathrm{i}}(\mathrm{fs})$ & $T_{1: \mathrm{s}}(\mathrm{ps})$ & $\tau_{\mathrm{s}}(\mathrm{ps})$ & $S$ & $R_{o}$ \\
\hline 303 & 15 & & & & & $8.3(4)$ & $0.65(1)$ & $0.357(2)$ \\
303 & 8 & $1.88^{\mathrm{a}}$ & $1.97^{\mathrm{a}}$ & $809(6)$ & $3.71(3)$ & $17.9(4)$ & $0.69(1)$ & $0.388(3)$ \\
303 & 5 & & & & & $28.0(3)$ & & $0.395(2)$ \\
313 & 5 & $1.95^{\mathrm{a}}$ & $1.71^{\mathrm{a}}$ & $831(25)$ & $3.53(7)$ & $12.4(2)$ & $0.51(1)$ & $0.375(2)$ \\
323 & 5 & $2.07^{\mathrm{a}}$ & $1.27^{\mathrm{a}}$ & $729(30)$ & $3.2(1)$ & $9.6(2)$ & & $0.389(4)$ \\
\hline \hline
\end{tabular}

${ }^{a} \mathrm{a}=$ fixed parameter

the reverse micelle $w_{o}$. This suggests that the surface water reorientation is curvature dependent. Smaller reverse micelles (stronger curvature) show slower surface reorientation.

The temperature-dependence of the reorientation time constant allows for an estimation of the activation energy of the reorientation of a water molecule at the surface by assuming an Arrhenius dependence. Figure 10 shows an Arrhenius plot of the reorientation rates for bulk water obtained from previous studies ${ }^{46}$ and of surface water. From least-squares fits values of $\Delta H_{\text {bulk }}^{\ddagger}=17.4 \pm 0.7 \mathrm{~kJ} \mathrm{~mol}^{-1}(4.15 \pm 0.16 \mathrm{kcal}$ $\left.\mathrm{mol}^{-1}\right)$ and $\Delta H_{\text {surface }}^{\ddagger}=45 \pm 9 \mathrm{~kJ} \mathrm{~mol}^{-1}\left(11 \pm 2 \mathrm{kcal} \mathrm{mol}^{-1}\right)$ were obtained. The large uncertainty is a result of the small number of data points. Our surface reorientation-rate measurements are, unfortunately, restricted to a small temperature range. For temperatures lower than $303 \mathrm{~K}$, the rate is too slow to be resolved whereas above $323 \mathrm{~K}$ the system reaches the solubilization limit boundary of the reverse micellar phase, at which water is expelled from the reverse micelles. This difference in $\Delta H^{\ddagger}$ values for the reorientation of bulk water and surface water suggests that the slower water reorientation on the surface is not only a result of reduced rotational freedom, i.e., an entropic effect, ${ }^{49}$ but is also a result of an increased enthalpy barrier.

From size-dependent anisotropy measurements, it can be observed that the amount of bulk water increases with the size of the reverse micelle. Since we know from the SAXS measurements the shape and geometry of the reverse micelles, and how their radius increases with $w_{\circ}$, we can estimate the layer thickness of the surface water. Taking a sphere of bulk water with a layer of surface-water of thickness $d$, one obtains the following expression for the fraction of bulk water $S$ with re-

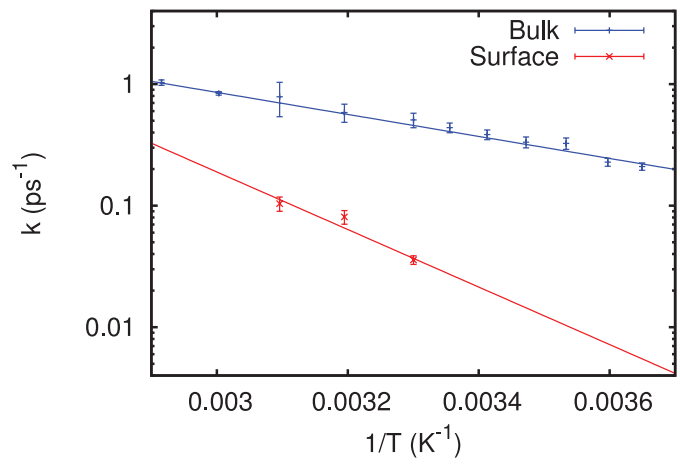

FIG. 10. Arrhenius plot of the reorientation rate of water versus reciprocal temperature. The red data points are from surface water and the blue are of bulk water taken from Ref. 46. spect to the total amount of water as function of radius $r$ (see Figure 12):

$$
\begin{aligned}
S & =\frac{\frac{4}{3} \pi(r-d)^{3}}{\frac{4}{3} \pi r^{3}} \\
& =1-3\left(\frac{d}{r}\right)+3\left(\frac{d}{r}\right)^{2}-\left(\frac{d}{r}\right)^{3} .
\end{aligned}
$$

The radius of a reverse micelle scales linearly with $w_{o}$ :

$$
r=a w_{o}
$$

and the bulk fraction can be written as a function of $w_{o}$ as

$$
S\left(w_{o}\right)=1-3\left(\frac{d}{a w_{o}}\right)+3\left(\frac{d}{a w_{o}}\right)^{2}-\left(\frac{d}{a w_{o}}\right)^{3} .
$$

Fitting the expression for $S\left(w_{o}\right)$ to the experimentally observed values of $S$ gives a value for $d / a$ of 1.1(3), see Figure 11. Using the proportionality constant $a=0.21(1) \mathrm{nm}$ obtained from the SAXS measurements, we can estimate that the thickness of the water layer is 2.4(4) $\AA$. This corresponds to the size of a water molecule $\left(2.8 \AA,^{50}\right.$ the O-O distance in liquid water) suggesting that only the outermost layer of water molecules are affected by confinement.

The conformation of the hydrophilic polyethylene oxide (PEO) chain in nonionic reverse micelles is still a matter of controversy. ${ }^{23,31,51,52}$ Numerous papers depict the structure of a nonionic reverse micelle. Mostly, they show

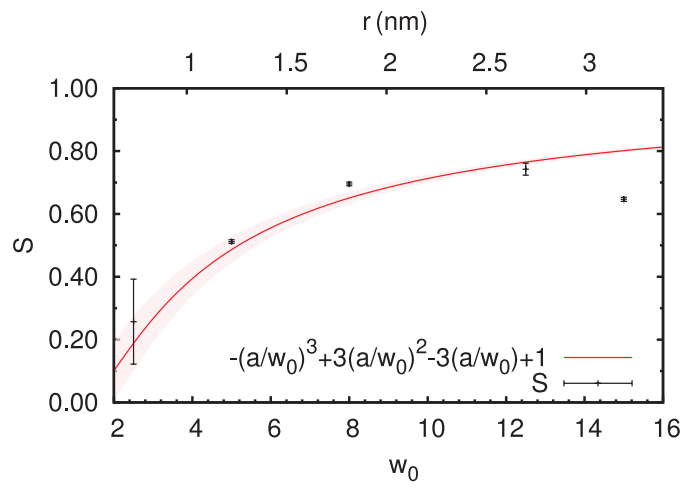

FIG. 11. Bulk water fraction $S$ versus $w_{\circ}$ and the micelle radius $r_{\circ}$. The data are fitted with the single-parameter function $S\left(w_{o}\right)$, see Eq. (11). The shaded area indicates the size-dependent uncertainty of the fit function $S\left(w_{o}\right)$ for one standard deviation in the fit parameter $\mathrm{d}$, as obtained by propagating the uncertainty of the fit parameter. $S$ for $w_{\circ}=2.5$ and 12.5 were analyzed separately using the lifetimes obtained from the global fit. 


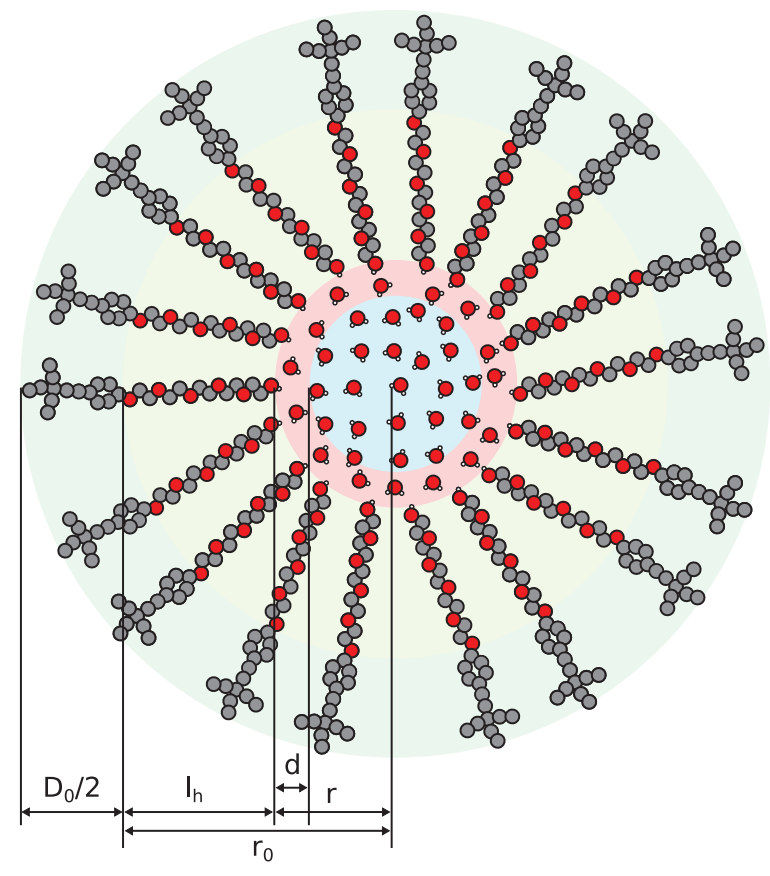

FIG. 12. Representation of the structure of an Igepal reverse micelle. The surfactant molecules form a saturated monolayer around the interior water droplets. Polyether glycol chains are stretched into zigzag configuration ${ }^{40}$ and do not or hardly penetrate the water volume. Oxygen, carbon, and hydrogen atoms are shown as black, red, and white dots, respectively.

that the PEO chain penetrates the water volume and dangles freely in the water body. ${ }^{31,52}$ We can test this hypothesis with our measurements: for reverse micelles with $w_{\text {。 }}$ $=5$, there are twice as many oxygen lone pairs available from the Igepal molecules as there are water molecules $(5$ ether groups per Igepal $\times 2$ number of lone pairs per ether $/ 5$ water molecules per Igepal). This would imply that if the hydrophilic chains would completely penetrate the reverse micellar interior, all water molecules would be perturbed. The interior of the reverse micelle would behave like an aqueous polymer solution due to the dangling PEO chains. From the anisotropy decay and the model that we use, we can conclude that this is definitely not the case. In contrast, we find a significant amount of unperturbed water that still behaves like bulk water. Other studies, in particular NMR experiments and vibrational energy relaxation of probe molecules, support this observation. ${ }^{23,51}$ From our study, we conclude that there is no water between the PEO chains, for these small reverse micelles as represented in Figure 12. However, we observe that for $w_{\circ}=15, S$ deviates a lot from the fit. This suggests that modeling the surface water as a layer on a sphere does not hold for large $w_{\circ}$. A possible explanation is that the polyether glycol chains are hydrated for large $w_{\circ}$ and therefore would give rise to a bigger surface water fraction. This hypothesis would have to be confirmed by further experiments. Nanocavities containing "pure" water were also found for water-tetraethyleneoxide binary mixtures at concentrations that are comparable to the small $w_{\circ}$ values. ${ }^{53}$

It is interesting to note that whereas the steady-state absorption spectrum shows a blue shift with decreasing micelle size, the transient spectra show no significant shift (see Figure 9). We believe this can be explained by a difference in the OD-stretch transition-dipole moments of water and Igepal. The total OD-stretch response arises from the ODstretch of Igepal at low frequency and HDO at higher frequency. Reducing the micelle size (by decreasing $w_{\circ}$ ) has two effects: an increase of the Igepal contribution (at low ODstretch frequency), and a blue shift of the water OD-stretch mode due to weakening of the hydrogen bonds in smaller micelles. ${ }^{41,47}$ These counteracting effects can lead to different net results in the steady-state (linear) absorption spectrum and the nonlinear transient absorption spectrum if the ODstretch transition-dipole moments $\mu_{\mathrm{OD}}$ of HDO and Igepal are different: in the linear spectrum the contributions of the two species (water and Igepal) scale with $\mu_{\mathrm{OD}}^{2}$, whereas in the nonlinear pump-probe spectrum they scale with $\mu_{\mathrm{OD}}^{4}$. It is difficult to determine $\mu_{\mathrm{OD}}$ experimentally for Igepal in the reverse micelles, because of overlap of the water and Igepal bands. Determining the Igepal transition-dipole moment from the integrated OD-stretch band of neat Igepal is not possible, because the average hydrogen-bond strength in neat Igepal is much less than in Igepal bound to water (as can be derived from the high OD-stretch frequency of neat Igepal, see Fig. 4). To get an estimate for that ratio of the water and Igepal OD-stretch transition-dipole moments in the reverse micelles, we can compare the experimentally determined $\mu_{\mathrm{OD}}$ of liquid $\mathrm{MeOD}$ and $\mathrm{HDO}$ in $\mathrm{H}_{2} \mathrm{O},{ }^{54,55}$ The ratio of these transitiondipole moments is $\mu_{\mathrm{OD}, \mathrm{MeOD}} / \mu_{\mathrm{OD}, \mathrm{HDO}}=13.9 / 9.3$. We may thus expect that Igepal contributes much more strongly (approximately 2.2 times as much) to the nonlinear spectrum than to the linear absorption spectrum. This will counteract the blue shift of the water contribution with decreasing micelle size, so that the micelle size will have less effect on the nonlinear spectrum than on the linear spectrum.

When comparing the steady-state spectrum of Igepal OD-stretch (Figure 4) with the Igepal contribution in the timeresolved data in the reverse micelles (Figure 9) a spectral shift is observed between the two. This discrepancy can be explained as follows. In neat Igepal, the OD group can only donate a hydrogen bond to another Igepal molecule. However in reverse micelles, Igepal can both accept and donate hydrogen bonds to neighboring water molecules. This difference in local environment strongly affects the vibration of the alcohol group. For example, the hydrogen bond acceptor-bound alcohol groups redshift only $100 \mathrm{~cm}^{-1}$ compared to monomer alcohols. However, acceptor and donator bound alcohol groups redshift $300 \mathrm{~cm}^{-1}$ compared to monomer alcohols. ${ }^{56}$

\section{CONCLUSION}

At low frequencies (around $2460 \mathrm{~cm}^{-1}$ ), we observe the response of the OD groups of the Igepal surfactant that shows a fast vibrational relaxation with a time constant of $800 \mathrm{fs}$. At a higher frequency of (around $2520 \mathrm{~cm}^{-1}$ ), we observe the response of both the bulk-like water and the surface-bound water showing vibrational relaxation time constants of 1.9 and 3.6 ps (at $300 \mathrm{~K}$ ), respectively. The reorientation data were modeled with a fast reorientation time constant $\tau_{b}$, set to the bulk value of water, and a fitted slow reorientation time constant $\tau_{s}$ which was assigned to surface water. From the sizeand temperature-dependent measurements, we conclude that 
the bulk water fraction is relatively large and, therefore, the PEO chains do not penetrate into the water volume. Since SAXS measurements confirmed the spherical nature of the reverse micelles and allow us to determine the proportionality factor between their interior water pool size and $w_{\circ}$, we show that the layer of slow reorienting surface water is approximately as thick as one water molecule (2.4(4) A).

The temperature-dependence of the surface reorientation time provides an estimate for the enthalpy barrier for the reorientation of surface water. We find $\Delta H_{\text {surface }}^{\ddagger}$ of $45 \pm 9 \mathrm{~kJ}$ $\mathrm{mol}^{-1}$, which is close to the barrier of ice at $54 \mathrm{~kJ} \mathrm{~mol}^{-1}$ (Ref. 57) and of water at protein surfaces. ${ }^{58}$ The dynamics of proteins are highly coupled to the dynamics of their surrounding water molecules. It was previously shown that water on reverse micellar surfaces, acting as model system for water around proteins, reorients slower than in the bulk..$^{29,47}$ Our results show that surface water reorientation is an activated process, and that the energy barrier is significantly larger than in bulk water. Therefore, the slowing down of surface water at the nonionic reverse micellar surface is not only an effect of a reduction of entropy, but also is also a result of a change in the enthalpy barrier. The more detailed picture of the surfactant/water interface (depicted in Figure 12) obtained from our experiments should deepen the understanding of many physical and chemical processes in neutral reverse micelles. Well known examples of such processes include the solubilization of proteins or other (ionic) species, and crystallization pathways in reverse micelles and their influence on the morphogenesis of nanocrystals.

\section{ACKNOWLEDGMENTS}

The authors would like to thank Stichting voor Fundamenteel Onderzoek der Materie (FOM) for financial support and Dr. P. Konarev (EMBL-Hamburg) for assistance in performing the SAXS experiments.

${ }^{1}$ R. Rand and V. Luzzati, Biophys. J. 8, 125 (1968).

${ }^{2}$ J. C. Rasaiah, S. Garde, and G. Hummer, Annu. Rev. Phys. Chem. 59, 713 (2008).

${ }^{3}$ G. Hummer, Mol. Phys. 105, 201 (2007).

${ }^{4}$ J. L. England, D. Lucent, and V. S. Pande, J. Am. Chem. Soc. 130, 11838 (2008).

${ }^{5}$ N. E. Levinger, Science 298, 1722 (2002).

${ }^{6}$ M.-P. Pileni, Nature Mater. 2, 145 (2003).

${ }^{7}$ W. Sager, "Microemulsion templating," in Nanostructured Soft Matter: Experiment, Theory, Simulation and Perspectives, edited by Zvelindovsky (Springer, Dordrecht, 2007), pp. 3-44.

${ }^{8}$ C. E. Nicholson, C. Chen, B. Mendis, and S. J. Cooper, Cryst. Growth Des. 11, 363 (2011).

${ }^{9}$ W. M. Gelbart, A. Ben-Shaul, and D. Roux, Micelles, Membranes, Microemulsions and Monolayers (Springer, Dordrecht, 1994).

${ }^{10}$ Statistical Thermodynamics of Surfaces, Interfaces and Membranes, edited by S. A. Safran (Addison-Wesley, Reading, 1994).

${ }^{11}$ E. M. Blokhuis and W. F. C. Sager, J. Chem. Phys. 110, 3148 (1999).

${ }^{12}$ E. M. Blokhuis and W. F. C. Sager, J. Chem. Phys. 111, 7062 (1999).

${ }^{13}$ E. M. Blokhuis and W. F. C. Sager, J. Chem. Phys. 115, 1073 (2001).

${ }^{14}$ M. Kahlweit, R. Strey, P. Firman, D. Haase, J. Jen, and R. Schomaecker, Langmuir 4, 499 (1988).

${ }^{15}$ W. M. Cox and J. H. Wolfenden, Proc. R. Soc. London, Ser. A 145, 475 (1934).

${ }^{16}$ R. Buchner, T. Chen, and G. Hefter, J. Phys. Chem. B 108, 2365 (2004).

${ }^{17}$ M. Kahlweit, R. Strey, and G. Busse, J. Phys. Chem. 94, 3881 (1990).

${ }^{18}$ J. Milano-Brusco, S. Prevost, D. Lugo, M. Gradzielski, and R. Schomacker, New J. Chem. 33, 1726 (2009).
${ }^{19}$ D. I. Svergun, P. V. Konarev, V. V. Volkov, M. H. J. Koch, W. F. C. Sager, J. Smeets, and E. M. Blokhuis, J. Chem. Phys. 113, 1651 (2000).

${ }^{20}$ M. Hirai, R. Kawai-Hirai, M. Sanada, H. Iwase, and S. Mitsuya, J. Phys. Chem. B 103, 9658 (1999).

${ }^{21}$ J. E. Boyd, A. Briskman, V. L. Colvin, and D. M. Mittleman, Phys. Rev. Lett. 87, 147401 (2001).

${ }^{22}$ B. Baruah, J. M. Roden, M. Sedgwick, N. M. Correa, D. C. Crans, and N. E. Levinger, J. Am. Chem. Soc. 128, 12758 (2006).

${ }^{23}$ Q. Zhong, A. P. Baronavski, and J. C. Owrutsky, J. Chem. Phys. 118, 7074 (2003).

${ }^{24}$ E. M. Corbeil and N. E. Levinger, Langmuir 19, 7264 (2003).

${ }^{25}$ N. E. Levinger and L. A. Swafford, Annu. Rev. Phys. Chem. 60, 385 (2009).

${ }^{26} \mathrm{C}$. Ronne and S. R. Keiding, "Molecular liquids: Water at the new millenium," J. Mol. Liq. 101, 199 (2002).

${ }^{27}$ A. M. Dokter, S. Woutersen, and H. J. Bakker, Proc. Natl. Acad. Sci. U.S.A. 103, 15355 (2006).

${ }^{28}$ A. M. Dokter, S. Woutersen, and H. J. Bakker, J. Chem. Phys. 126, 124507 (2007).

${ }^{29}$ I. R. Piletic, D. E. Moilanen, D. B. Spry, N. E. Levinger, and M. D. Fayer, J. Phys. Chem. A 110, 4985 (2006).

${ }^{30}$ E. E. Fenn, D. B. Wong, and M. D. Fayer, Proc. Natl. Acad. Sci. U.S.A. 106, 15243 (2009).

${ }^{31}$ D. E. Moilanen, N. E. Levinger, D. B. Spry, and M. D. Fayer, J. Am. Chem. Soc. 129, 14311 (2007).

${ }^{32}$ S. Park, D. E. Moilanen, and M. D. Fayer, J. Phys. Chem. B 112, 5279 (2008).

${ }^{33}$ C. Ronne, P. O. Astrand, and S. R. Keiding, Phys. Rev. Lett. 82, 2888 (1999).

${ }^{34}$ Y. L. A. Rezus and H. J. Bakker, J. Chem. Phys. 123, 114502 (2005).

${ }^{35}$ M. W. Roessle, R. Klaering, U. Ristau, B. Robrahn, D. Jahn, T. Gehrmann, P. Konarev, A. Round, S. Fiedler, C. Hermes, and D. Svergun, J. Appl. Crystallogr. 40, s190 (2007).

${ }^{36}$ A. Vrij, J. Chem. Phys. 71, 3267 (1979).

${ }^{37}$ D. Gazzillo and A. Giacometti, J. Chem. Phys. 113, 9837 (2000).

${ }^{38}$ T. Vad, H.-G. Haubold, N. Waldofner, and H. Bonnemann, J. Appl. Crystallogr. 35, 459 (2002).

${ }^{39}$ H. Graener, G. Seifert, and A. Laubereau, Chem. Phys. Lett. 172, 435 (1990).

${ }^{40}$ V. Degiorgio, "Non-ionic micelles," in Physics of Amphiphiles: Micelles, Vesicles and Microemulsions, edited by V. Degiorgio (North-Holland, 1985), pp. 303-332.

${ }^{41}$ T. Podlipskaya, A. Bulavchenko, and L. Sheludyakova, J. Struct. Chem. 48, 236 (2007).

${ }^{42}$ A. Novak, Structure and Bonding (Springer, Berlin/Heidelberg, 1974), Vol. 18, pp. 177-216.

${ }^{43}$ G. Seifert, T. Patzlaff, and H. Graener, Phys. Rev. Lett. 88, 147402 (2002).

${ }^{44}$ D. J. Shaw, M. R. Panman, and S. Woutersen, Phys. Rev. Lett. 103, 227401 (2009).

${ }^{45}$ K. J. Gaffney, P. H. Davis, I. R. Piletic, N. E. Levinger, and M. D. Fayer, J. Phys. Chem. A 106, 12012 (2002).

${ }^{46}$ K. Tielrooij, C. Petersen, Y. Rezus, and H. Bakker, Chem. Phys. Lett. 471, 71 (2009).

${ }^{47}$ A. M. Dokter, S. Woutersen, and H. J. Bakker, Phys. Rev. Lett. 94, 178301 (2005).

${ }^{48}$ J. J. Loparo, C. J. Fecko, J. D. Eaves, S. T. Roberts, and A. Tokmakoff, Phys. Rev. B 70, 180201 (2004).

${ }^{49}$ D. Laage, G. Stirnemann, F. Sterpone, R. Rey, and J. T. Hynes, Annu. Rev. Phys. Chem. 62, 395 (2011).

${ }^{50}$ A. H. Narten, M. D. Danford, and H. A. Levy, Discuss. Faraday Soc. 43, 97 (1967).

${ }^{51}$ M. A. Sedgwick, D. C. Crans, and N. E. Levinger, Langmuir 25, 5496 (2009).

${ }^{52}$ J.-L. Lemyre, S. Lamarre, A. Beaupre, and A. M. Ritcey, Langmuir 26, 10524 (2010).

${ }^{53}$ E. E. Fenn, D. E. Moilanen, N. E. Levinger, and M. D. Fayer, J. Am. Chem. Soc. 131, 5530 (2009).

${ }^{54}$ J. E. Bertie and S. L. Zhang, J. Mol. Struct. 413, 333 (1997).

${ }^{55}$ J. E. Bertie, M. K. Ahmed, and H. H. Eysel, J. Phys. Chem. 93, 2210 (1989).

${ }^{56}$ U. Liddel and E. D. Becker, Spectrochimica Acta 10, 70 (1957).

${ }^{57}$ R. J. Wittebort, M. G. Usha, D. J. Ruben, D. E. Wemmer, and A. Pines, J. Am. Chem. Soc. 110, 5668 (1988).

${ }^{58}$ S. Fischer, C. S. Verma, and R. E. Hubbard, J. Phys. Chem. B 102, 1797 (1998). 